\title{
Bitter melon fruit extract affects hepatic expression of the genes involved in inflammation and fatty acid metabolism in $o b / o b$ mice
}

\author{
Dinia Rizqi Dwijayanti' ${ }^{1}$, Tetsuya Okuyama ${ }^{1}$, Toshinari Ishii ${ }^{1}$, Eri Mukai ${ }^{2}$, and Mikio \\ Nishizawa ${ }^{1}$
}

\begin{abstract}
${ }^{1}$ Medical Chemistry Laboratory, Department of Biomedical Sciences, College of Life Sciences, Ritsumeikan University, Kusatsu, Shiga, Japan, ${ }^{2}$ Medical Physiology and Metabolism Laboratory, Department of Biomedical Sciences, College of Life Sciences, Ritsumeikan University, Kusatsu, Shiga, Japan
\end{abstract}

Corresponding author: Mikio Nishizawa, M.D., Ph.D., Department of Biomedical Sciences, College of Life Sciences, Ritsumeikan University, 1-1-1 Nojihigashi, Kusatsu, Shiga 525-8577, Japan

Submission Date: November 13, 2019 Acceptance Date: January 25 ${ }^{\text {th }}$, 2020 Publication Date: January $31^{\text {st }}, 2020$

Citation: Dwijayanti D.R., Okuyama T., Ishii T., Mukai E., and Nishizawa M. Bitter melon fruit extract affects hepatic expression of the genes involved in inflammation and fatty acid metabolism in ob/ob mice. Functional Foods in Health and Disease 2020; 10(1): 18-36. DOI: https:/doi.org/10.31989/ffhd.v10i1.675

\begin{abstract}
Background: The fruit of bitter melon (Momordica charantia L.) has traditionally been used as a crude drug to treat obesity and diabetes mellitus. Previously, we reported that an ethyl acetate (EtOAc)-soluble fraction of bitter melon fruit extract showed anti-inflammatory effects on interleukin-1 $\beta$-treated rat hepatocytes. The liver plays a pivotal role to maintain homeostasis of glucose and lipid. It is speculated that the administration of an EtOAc-soluble fraction of a bitter melon fruit extract (BMFA) may change the expression profile of hepatic genes involved in inflammation, glucose metabolism, and lipid metabolism.
\end{abstract}

Objective: We aimed to evaluate the effects of BMFA on the gene expression in the liver of $o b / o b$ mice, which are deficient in leptin and are used as a diabetes mellitus model. The changes may be related to hepatic steatosis and obesity.

Methods: Male 8-week-old $o b / o b$ mice (average body weight, $40.9 \mathrm{~g}$ ) were fed a standard diet with and without BMFA (ob/ob+BMFA and $o b / o b-B M F A$ groups, respectively; 4 mice/group) for seven days. The livers were excised to prepare total RNA for microarray expression analyses. Signal ratios (i.e., ob/ob+BMFA versus ob/ob-BMFA group) were calculated to compare gene expression. 
Results: The mRNA levels of 2,632 genes were significantly changed in the livers of $o b / o b+\mathrm{BMFA}$ mice when compared with those of ob/ob-BMFA mice. As expected, proinflammatory cytokine and chemokine genes were significantly downregulated by BMFA. The acyl-Coenzyme A thioesterase 3 gene in fatty acid oxidation was upregulated in $o b / o b+\mathrm{BMFA}$ liver, whereas free fatty acid receptor 2 and solute carrier family 27 member 3 genes were downregulated. Most genes involved in glycolysis and gluconeogenesis were not affected by BMFA. In contrast, the level of peroxisome proliferator-activated receptor $\gamma$ coactivator $1 \alpha$ mRNA significantly increased in $o b / o b+\mathrm{BMFA}$ mice, suggesting that BMFA administration may reduce insulin resistance in $o b / o b$ mice.

Conclusion: Our study suggested that BMFA may possess anti-inflammatory effects and enhance fatty acid metabolism in $o b / o b$ mouse liver. These data imply the possibility that EtOAc-soluble constituents in bitter melon fruit may improve lipid accumulation in the liver during obesity and diabetes mellitus, as well as non-alcoholic fatty liver disease.

Keywords: Momordica charantia, gene expression, inflammation, diabetes mellitus, nonalcoholic fatty liver disease.

\section{INTRODUCTION}

Momordica charantia L. (Cucurbitaceae) is known as bitter melon and is cultivated in tropical and subtropical regions, including Asian countries, such as Indonesia and Japan. The fruit of bitter melon has traditionally been used as a crude drug to treat obesity and diabetes mellitus. Supplementation with bitter melon reduced the body weight of mice that were fed a high-fat diet possibly by increasing lipid oxidation [1]. Regarding the hypolipidemic and hypoglycemic effects of bitter melon extracts, several mechanisms are speculated: increase in glucose uptake in the liver, facilitation of glucose metabolism, and enhancement of insulin action [2].

Obesity is a condition in which energy intake is higher than energy expenditure and is characterized by excessive accumulation of lipid in the body, which increases body weight [3]. A systematic review and meta-analysis showed that significant associations with obesity and overweight were found with the incidence of type 2 diabetes mellitus, as well as cancer and cardiovascular diseases [4]. Dyslipidemia is commonly observed in diabetes mellitus patients, and there is strong evidence that cholesterol lowering improves cardiovascular outcomes [5]. In addition, high-fat feeding of rats increased mRNA levels of leptin, but not fatty acid synthase, in adipose tissue and increased oxidative stress in the liver [6]. Leptin binds to its receptor to regulate the balance between food intake and energy expenditure [7].

The liver comprises hepatocytes, non-hepatocytic parenchymal cells, and various immune cells. The hepatocytes primarily maintain systemic homeostasis of glucose, and hepatic glucose production (ca. 90\% of endogenous glucose production) is crucial for glucose homeostasis [8]. Glucose is incorporated from plasma into hepatocytes and metabolized by glycolysis and the citric acid (Krebs) cycle. As shown in Figure 1, glucose homeostasis is maintained by glycolysis, gluconeogenesis, glycogenolysis, glycogen synthesis, and other pathways in hepatocytes [8]. Glucose is converted to glycogen, which is stored as an energy source.

Systemic homeostasis of lipid is also maintained by hepatocytes. Triglyceride (i.e., triacylglycerol) is stored at a content of less than 5\% of the liver as an energy source [9]. The steady state is attained by precisely regulated input (uptake from plasma and de novo synthesis) and output (degradation by oxidation and secretion into plasma) of fatty acid [9]. Fatty acids, as well as cholesterol, are incorporated into hepatocytes and degraded, de novo synthesized, or 
reused. Triglycerides are secreted into plasma as triglyceride-enriched very low-density lipoprotein (VLDL) [9].

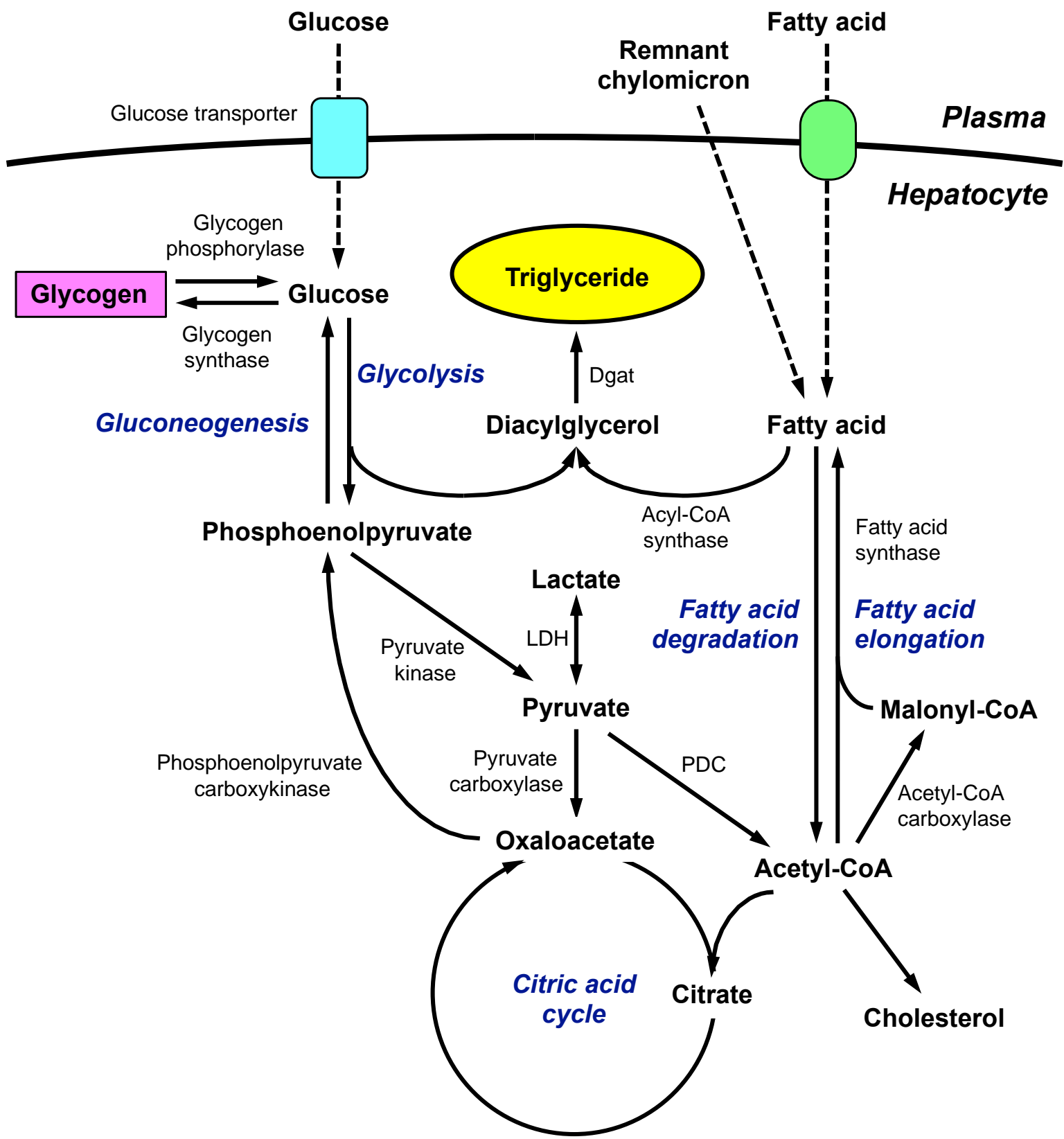

Figure 1. Overview of the pathways involved in the metabolism of glucose and fatty acid and the synthesis of glycogen and triglyceride in a hepatocyte. Glucose is incorporated from plasma into a hepatocyte through the glucose transporter (GLUT2, also known as solute carrier family 2 member 2), metabolized, and finally stored as glycogen [8]. Fatty acid that is conjugated with albumin in the blood is incorporated by the complex of fatty acid translocase/CD36, plasma membrane fatty acid-binding protein, and caveolin-1 [9]. Alternatively, chylomicron remnant is internalized into the cell, and fatty acid is de novo synthesized from acetyl-CoA. Diacylglycerol is synthesized from fatty acid and glycerol-3-phosphate (in glycolysis) and then converted to triglyceride (= triacylglycerol) by diacylglycerol $O$-acyltransferase (Dgat). Finally, triglyceride is stored in a lipid droplet [9]. Only the main reactions and enzymes are shown for simplicity (See details in the text). Each solid arrow indicates one or more reactions. Dashed arrows indicate the translocation of molecules. LDH, lactate dehydrogenase; PDC, pyruvate dehydrogenase complex. 
During inflammation, hepatocytes and Kupffer cells (resident macrophages in the liver) play an important role and produce a variety of proinflammatory mediators: nitric oxide (NO), chemokines, and proinflammatory cytokines, such as tumor necrosis factor (TNF) [10-12]. The transcription factor, nuclear factor $(\mathrm{NF})-\mathrm{kB}$ mediates the production of many of these mediators $[10,13]$. We previously reported that the ethyl acetate-soluble fraction of fruit extracts from bitter melons cultivated in Indonesia and Japan showed anti-inflammatory effects on interleukin (IL)-1 $\beta$-treated rat hepatocytes [14]. This hydrophobic fraction from the fruit extracts of both Indonesian and Japanese bitter melons comparably suppressed IL-1 $\beta$-induced NO production and the expression of proinflammatory cytokine mRNAs [14]. In this fraction, cucurbitane-type triterpenoids are assumed to be present. It is possible that the ethyl acetatesoluble fraction affects the genes that are involved in glucose and fatty acid metabolism in the liver.

The liver is affected by obesity and diabetes mellitus because it plays a key role in the metabolism of glucose and lipid. Under obesity and diabetic conditions, net glucose production is changed in hepatocytes by the dysregulation of the glucose and glycogen pathways, resulting in hepatic insulin resistance and fasting hyperglycemia [8]. Overnutrition and obesity causes dysregulation of lipid metabolism, leading to accumulation of triglycerides in the liver (i.e., hepatic steatosis), which is observed in diabetes mellitus and non-alcoholic fatty liver disease (NAFLD) [9]. Therefore, genes related to the metabolism and biosynthesis of glucose and fatty acid may be affected in hepatocytes. On the other hand, it is reported that proinflammatory cytokines and adipokines provoke immune responses and activate metabolic pathways in the liver and adipose tissue, leading to the development of insulin resistance $[15,16]$.

The $L e p^{o b / o b}$ mice are deficient in leptin and show hyperphagia and obesity [17]. Therefore, $L e p^{o b / o b}$ mice have been used as a type II diabetes mellitus model to examine antidiabetic effects. In this study, the ethyl acetate-soluble fraction of bitter melon fruit extract was orally administered to $L e p^{o b / o b}$ mice, and the short-term effects of this fraction on gene expression in the liver were examined and discussed.

\section{MATERIALS AND METHODS}

\section{Materials}

Methanol, ethyl acetate (EtOAc), and the Sepasol I Super G Solution were purchased from Nacalai Tesque Inc., Kyoto, Japan. A $\gamma$-ray-irradiated CRF-1 Certified Diet (according to Charles River' s formula) was obtained from Oriental Yeast Co., Ltd. (Tokyo, Japan) and used as a standard diet for mice. Quality of CRF-1 diet was certified by Charles River Laboratories Japan, Inc. (Yokohama, Japan), and its nutrient composition was: moisture, $8.5 \mathrm{~g}$; crude protein, $22.1 \mathrm{~g}$; crude fat, $5.4 \mathrm{~g}$; crude ash, $6.2 \mathrm{~g}$; crude fiber, $3.1 \mathrm{~g}$; nitrogen free extract, $54.6 \mathrm{~g}$; and calories, $355.8 \mathrm{kcal}$ (mean values per $100 \mathrm{~g}$ diet) (data kindly provided by Oriental Yeast Co., Ltd.). A Polytron homogenizer, model PT 1300D (Kinematica AG, Luzern, Switzerland) was used to extract total cellular RNA. An RNAqueous Kit and a TURBO DNA-free Kit were purchased from Applied Biosystems (Austin, TX, USA). A TapeStation system, a Low Input Quick Amp Labeling Kit, and SurePrint G3 Mouse Gene Expression 8×60K Microarrays ver. 2 were obtained from Agilent Technologies (Santa Clara, CA, USA). A SureScan Microarray Scanner G2600D (Agilent Technologies) and Feature Extraction Software, version 12.1.0.3 (Agilent Technologies) were used for the scanning of microarray slides and the analysis of the scanned data, respectively. 


\section{Preparation of a bitter melon fruit extract and fractionation}

The seeds of immature fruit of bitter melon (collected in Okinawa, Japan) were removed, and the fruit was extracted with methanol [14]. The resultant extract was fractionated into three crude fractions by hydrophobicity, as previously published [14]. The EtOAc-soluble fraction of a bitter melon fruit extract was evaporated and used for the subsequent experiments.

\section{Animals}

All animal care and experimental procedures were performed in accordance with the guidelines and laws of the Japanese Government and were approved by the Animal Care Committee of Ritsumeikan University, Biwako-Kusatsu Campus (No. BKC2018-029). Specific pathogenfree male $\mathrm{Lep}^{o b / o b}$ mice (hereafter, ob/ob mice) at the age of 7 weeks (Japan SLC, Inc., Hamamatsu, Japan) were housed at $21-23{ }^{\circ} \mathrm{C}$ under a 12 -h light-dark cycle with a $\gamma$-rayirradiated CRF-1 diet and water available ad libitum. The mice were acclimated for one week before the experiment.

\section{Administration of the EtOAc-soluble fraction of bitter melon fruit extract (BMFA) to mice}

The $o b / o b$ mice were randomly assigned into two groups (four mice per group) on Day 0 . The body weight (mean \pm standard deviation) was $40.2 \pm 0.90 \mathrm{~g}$ (ob/ob+BMFA mice) and $41.6 \pm$ $1.29 \mathrm{~g}$ (ob/ob-BMFA mice) on Day 0. Then, a CRF-1 diet was fed daily with and without $1 \%$ (of the weight of the diet) EtOAc-soluble fraction (ob/ob+BMFA and $o b / o b-\mathrm{BMFA}$ groups, respectively) for seven days. The intake of the diet was $4.05 \pm 0.50 \mathrm{~g} / \mathrm{mouse} / \mathrm{day}(o b / o b+\mathrm{BMFA}$ group) and $4.73 \pm 0.57 \mathrm{~g} / \mathrm{mouse} /$ day (ob/ob-BMFA group). Water was available ad libitum.

\section{Extraction of total cellular RNA from the liver}

On Day 7, the mice were euthanized by cervical dislocation. After excision of the liver, the tissue specimen was lysed in the Sepasol I Super G Solution using a Polytron homogenizer. Total cellular RNA was purified according to the previously published methods $[18,19]$. Briefly, this lysate was centrifugated at $10,000 \times g$ at $23{ }^{\circ} \mathrm{C}$ for $5 \mathrm{~min}$ to remove debris. After the addition of chloroform to the supernatant and centrifugation at $10,000 \times g$ at $23{ }^{\circ} \mathrm{C}$ for 15 min, total RNA was extracted from the aqueous layer, recovered by isopropanol precipitation, and purified with an RNAqueous Kit. Genomic DNA in the RNA samples was removed by a TURBO DNA-free kit. The quality of the total RNA was checked by native $1.5 \%$ agarose gel electrophoresis in TAE buffer (40 mM Tris-acetate, $\mathrm{pH} 8.0$, and $0.2 \mathrm{mM}$ EDTA) to confirm that there was no degradation of RNA samples.

\section{Microarray analyses of $m R N A$ expression}

The total RNA from the mouse liver was pooled in each group ( $n=4$ mice per group). The quality of the RNA sample was verified by automated electrophoresis using a TapeStation System and confirmed that these samples were able to be used for microarray analyses. The two samples were labeled with cyanine3 (Cy3) using the Low Input Quick Amp Labeling Kit. The resultant Cy3-labeled complementary RNA (cRNA) was fragmented and subjected to hybridization to the SurePrint G3 Mouse Gene Expression $8 \times 60 \mathrm{~K}$ Microarray ver. 2, according to the manufacturer's protocol. Each microarray contained 59,305 probe sets. After washing, the slides were scanned on the SureScan Microarray Scanner G2600D using one color scan. 
The scanned images were analyzed with Feature Extraction Software using default parameters to obtain background subtracted and spatially detrended processed signal intensities. Processed signal intensities were normalized by the global scaling method. A trimmed mean probe intensity was determined by removing $2 \%$ of the lower and the higher end of the probe intensities to calculate the scaling factor. Normalized signal intensities were then calculated from the target intensity on each array using the scaling factor so that the trimmed mean target intensity of each array was arbitrarily set to 2,500 .

\section{Evaluation of the data of microarray analyses}

Each probe set gave two signals derived from Cy3-labeled cRNA from ob/ob+BMFA mice ('Experiment' signal) and ob/ob-BMFA mice ('Base' signal). The signal was evaluated and scored as 'signal evaluation' by the Feature Extraction Software: signal evaluation $=2$ (signal detected), 1 (low signal intensity or detection unreliable), and 0 (signal not detected). When both the Experiment and Base signals of a probe set were detected (i.e., signal evaluation =2), this probe set was further analyzed. When either signal of a probe set was evaluated as 2 and the other was 1 or 0 , this probe set was carefully evaluated with its signals. In the other cases, the signal data of probe sets were excluded from the further analyses. When both the Experiment and Base signals of a probe set were very low $(<100)$ regardless of the signal evaluation, this probe set was excluded from the tables. Only the probe sets with reliable signals were selected to prepare tables. The probe sets were classified by Kyoto Encyclopedia of Genes and Genomes (KEGG) Pathway (https://www.genome.jp/kegg/pathway.html).

Experiment-to-Base signal ratios were calculated by comparing each probe set of the $o b / o b+\mathrm{BMFA}$ mice to the corresponding probe set the $o b / o b-\mathrm{BMFA}$ mice. Therefore, the signal ratio of BMFA (+) versus $(-)$ is the ratio of the Experiment signal divided by the Base signal and presented as the fold change of BMFA (+) to $(-)$. The significantly decreased transcripts were selected as probe sets with a signal ratio $\leq 0.5$, and the significantly increased transcripts were selected as probe sets with a signal ratio $\geq 2.0$. The probe sets that were significantly affected by BMFA administration were shown in the tables. Several probe sets that were not significantly affected were omitted from the tables.

\section{RESULTS}

\section{Profiles of mRNA expression in the livers of ob/ob mice}

To investigate the genome-wide profiles of mRNA expression affected by the EtOAc-soluble constituents in bitter melon fruit, microarray expression analyses $(59,305$ probe sets per microarray) were performed using total cellular RNA from the livers of $o b / o b$ mice ( $n=4$ per group). After a signal evaluation described in the Materials and Methods, a total of 32,731 probe sets were selected as the genes were expressed in the liver. (Although a single gene may have more than one probe set on the microarray, we hereafter use 'gene' instead of 'probe set' to easily understand.) The genes expressed in $o b / o b$ mouse liver (32,731 genes) are plotted in Figure 2. Then, signal ratios (i.e., ob/ob+BMFA versus ob/ob-BMFA) were calculated according to the Materials and Methods. We selected 2,632 genes whose mRNA levels were significantly changed by the administration of BMFA to ob/ob mice. Among these genes, the expression of 936 genes ( $2.9 \%$ of the genes expressed) was significantly increased (pink dots), 
whereas that of 1,696 genes (5.2\% of the genes expressed) was significantly decreased (blue dots) in the liver of $o b / o b+\mathrm{BMFA}$ mice compared to that of $o b / o b-\mathrm{BMFA}$ mice.

Next, we analyzed these genes that were significantly affected by BMFA. According to the classification by KEGG Pathway, we examined all the genes that are related to inflammation (cytokines and chemokines), fatty acid pathways, glucose pathways, glycogen pathways, and insulin resistance.

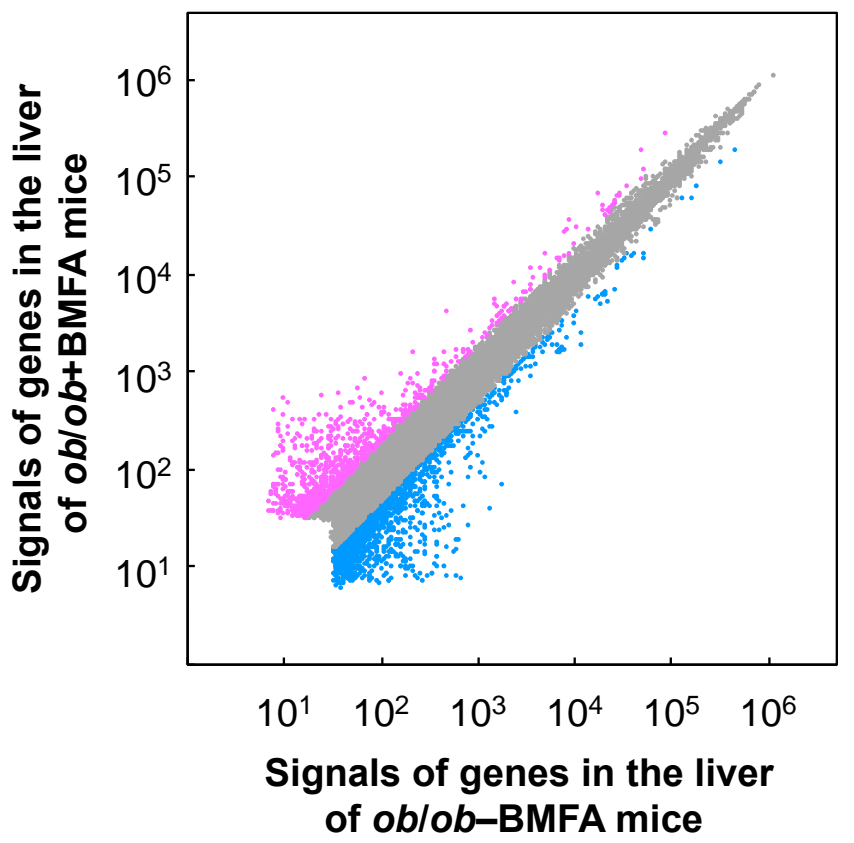

Figure 2. Gene expression in the livers of the $o b / o b$ mice administered the EtOAc-soluble fraction of BMF extract (BMFA). The $o b / o b$ mice were fed a standard diet daily with and without BMFA (ob/ob+BMFA and $o b / o b-\mathrm{BMFA}$ group, respectively), and the total RNA obtained from the liver of the two groups was subjected to microarray analyses. The genes expressed in $o b / o b$ mouse livers (32,731 probe sets) were plotted. Each probe set was plotted as a dot by its signal on a logarithmic scale. The pink dots represented genes that were significantly increased by BMFA (signal ratio $\geq 2.0$ ); gray dots represented genes whose expression was not changed $(0.5<$ signal ratio $<2.0)$; and blue dots represented genes that were significantly decreased by BMFA (signal ratio $\leq 0.5$ ).

\section{Effects of BMFA on the mRNA expression of proinflammatory genes in ob/ob mouse liver}

First, we investigated the genes involved in inflammation. The probe sets classified as 'Cytokine-cytokine receptor interaction' by KEGG Pathway were analyzed. As shown in Table 1 , IL-1 $\beta$ significantly decreased in $o b / o b+B M F A$ mouse liver. The expression levels of mRNAs encoding the TNF receptor superfamily (Tnfrsf10b and Tnfrsf22) and Toll-like receptor (TLR) 1 and 2 decreased.

In contrast, the genes induced by acute inflammation in hepatocytes or Kupffer cells $[19,20]$ were absent or not affected by BMFA. Cyclooxygenase-2 (COX-2, also known as prostaglandin-endoperoxide synthase 2, Ptgs2) mRNA was not detected in the liver of $o b / o b$ mice (data not shown). The mRNA expression levels of nitric oxide synthase 2 (NOS2; also known as iNOS), TNF, C-reactive protein (CRP), and lipocalin 2 (LCN2) were not 
significantly changed in $o b / o b+\mathrm{BMFA}$ mice. CRP and LCN2 are acute-phase proteins and biomarkers of inflammation and infection [21,22].

Table 1. Hepatic expression of mRNAs encoding cytokines and acute-phase proteins.

\begin{tabular}{llc}
\hline Description (Abbreviation) & $\begin{array}{l}\text { Gene } \\
\text { symbol }\end{array}$ & $\begin{array}{l}\text { Signal ratio of } \\
\text { BMFA(+) vs. }(-)^{*}\end{array}$ \\
\hline Decreased: & & \\
Interleukin-1 $\beta$ (IL-1 $\beta$ ) & Illb & 0.278 \\
Tumor necrosis factor receptor superfamily, member 10b & Tnfrsf10b & 0.291 \\
Toll-like receptor 1 (TLR1) & Tlr1 & 0.317 \\
Toll-like receptor 2 (TLR2) & Tlr2 & 0.331 \\
Tumor necrosis factor receptor superfamily, member 22 & Tnfrsf22 & 0.410 \\
\hline Not changed: & & \\
\hline Lipocalin 2 (LCN2) & Lcn2 & 0.546 \\
Colony stimulating factor 2 receptor $\beta$, low-affinity & Csf2rb & 0.594 \\
Mus musculus colony stimulating factor 1 (macrophage) & Csf 1 & 0.695 \\
Tumor necrosis factor (TNF) & Tnf & 0.774 \\
C-reactive protein, pentraxin-related (CRP) & Crp & 0.974 \\
Nitric oxide synthase 2 (NOS2, iNOS) & Nos 2 & 1.014 \\
\hline
\end{tabular}

${ }^{*}$ Signal ratios of probe sets in $o b / o b+\mathrm{BMFA} v s$. $o b / o b-\mathrm{BMFA}$ are expressed as the fold change, when the signal of $o b / o b$-BMFA was set to 1 .

Second, ligands and their receptors of chemokines ('Chemokine signaling pathway' classified by KEGG Pathway) were examined. Similar to proinflammatory cytokine genes, BMFA downregulated the expression of many chemokine genes (Table 2). BMFA decreased the levels of mRNAs encoding chemokine C-C motif ligand mRNAs (CCL2, CCL3, CCL4, CCL6, and CCL7) and C-X-C motif ligand 10 (CXCL10) in the liver of $o b / o b$ mice.

Table 2. Hepatic expression of chemokine mRNAs.

\begin{tabular}{llc}
\hline Description (Abbreviation) & $\begin{array}{l}\text { Gene } \\
\text { symbol }\end{array}$ & $\begin{array}{l}\text { Signal ratio of } \\
\text { BMFA(+) vs. }(-)^{*}\end{array}$ \\
\hline Decreased: & Ccl7 & 0.139 \\
Chemokine C-C motif chemokine ligand 7 (CCL7) & Ccl2 & 0.167 \\
Chemokine C-C motif ligand 2 (CCL2) & Ccl3 & 0.239 \\
Chemokine C-C motif ligand 3 (CCL3) & Cxcllo & 0.334 \\
Chemokine C-X-C motif ligand 10 (CXCL10) & $C c l 4$ & 0.356 \\
Chemokine C-C motif ligand 4 (CCL4) & $C c l 6$ & 0.429 \\
Chemokine C-C motif ligand 6 (CCL6) & $C x 3 c r 1$ & 0.445 \\
Chemokine C-X 3 -C motif receptor 1 (CX3CR1) & $C c r 7$ & 0.487 \\
Chemokine C-C motif receptor 7 (CCR7) & .
\end{tabular}

${ }^{*}$ Signal ratios of probe sets in $o b / o b+\mathrm{BMFA} v s . o b / o b-\mathrm{BMFA}$ are expressed as the fold change, when the signal of $o b / o b-\mathrm{BMFA}$ was set to 1 .

\section{Effects of BMFA on the mRNA expression of enzymes that catalyze fatty acid biosynthesis and metabolism}

Third, the genes related to fatty acid biosynthesis ('Fatty acid biosynthesis' and 'Fatty acid elongation' classified by KEGG Pathway) were analyzed. Acetyl-Coenzyme A (CoA) 
carboxylase, which consists of $\alpha$ subunit (Acaca) and $\beta$ subunit (Acacb), converts acetyl-CoA to malonyl-CoA. Then, acyl-acyl carrier protein (ACP) is elongated by cycling through a fourstep process (condensation, reduction, dehydration, and reduction). Fatty acid synthase (Fasn, type I FAS) catalyzes all these reactions in cytosol. As shown in Table 3, BMFA administration did not change the expression of the Acaca, Acacb, or Fasn genes.

Table 3. Hepatic expression of mRNAs involved in fatty acid biosynthesis

\begin{tabular}{|c|c|c|}
\hline Gene name (Abbreviation) & $\begin{array}{l}\text { Gene } \\
\text { symbol }\end{array}$ & $\begin{array}{l}\text { Signal ratio of } \\
\operatorname{BMFA}(+) v s .(-)^{*}\end{array}$ \\
\hline \multicolumn{3}{|l|}{ Decreased: } \\
\hline ELOVL family member 7, elongation of long chain fatty acids & Elovl7 & 0.146 \\
\hline Free fatty acid receptor 2 & Ffar2 & 0.211 \\
\hline $\begin{array}{l}\text { Solute carrier family } 27 \text { (fatty acid transporter), member } 3 \\
\text { (FATP3) }\end{array}$ & Slc27a3 & 0.466 \\
\hline \multicolumn{3}{|l|}{ Not changed: } \\
\hline Solute carrier family 27 (fatty acid transporter), member 4 & Slc27a4 & 0.633 \\
\hline Acetyl-Coenzyme A (CoA) carboxylase, alpha subunit & Acaca & 0.661 \\
\hline ELOVL family member 1 , elongation of long chain fatty acids & Elovl1 & 0.747 \\
\hline Fatty acid synthase (type I FAS) & Fasn & 0.755 \\
\hline Glycerol-3-phosphate acyltransferase, mitochondrial & Gpam & 0.804 \\
\hline Hydroxysteroid (17-beta) dehydrogenase 12 & Hsd17b12 & 0.901 \\
\hline Mitochondrial trans-2-enoyl-CoA reductase & Mecr & 0.918 \\
\hline ATP citrate lyase & Acly & 0.942 \\
\hline trans-2,3-Enoyl-CoA reductase & Tecr & 0.976 \\
\hline 3-Hydroxyacyl-CoA dehydratase 2 & Hacd 2 & 1.030 \\
\hline 3-Hydroxyacyl-CoA dehydratase 3 & Hacd3 & 1.062 \\
\hline Malonyl CoA-ACP acyltransferase, mitochondrial & Mcat & 1.071 \\
\hline Acetyl-CoA carboxylase, beta subunit & $A c a c b$ & 1.079 \\
\hline Diacylglycerol $O$-acyltransferase 2 & Dgat2 & 1.143 \\
\hline Diacylglycerol $O$-acyltransferase 1 & Dgat1 & 1.154 \\
\hline Carbonyl reductase 4 & Cbr4 & 1.265 \\
\hline Stearoyl-CoA desaturase 1 & Scdl & 1.265 \\
\hline 3-Oxoacyl-ACP synthase, mitochondrial & Oxsm & 1.284 \\
\hline Solute carrier family 27 (fatty acid transporter), member 2 & Slc27a2 & 1.299 \\
\hline ELOVL family member 6 , elongation of long chain fatty acids & Elovl6 & 1.346 \\
\hline
\end{tabular}

${ }^{*}$ Signal ratios of probe sets in $o b / o b+\mathrm{BMFA} v s$. $o b / o b-\mathrm{BMFA}$ are expressed as the fold change, when the signal of $o b / o b-$ BMFA was set to 1 .

In mitochondria, malonyl-CoA-ACP acyltransferase, mitochondrial (Mcat) synthesizes malonyl-ACP from acetyl-CoA. Then, type II FAS complex, which consists of four mitochondrial enzymes, elongates acyl-ACP by cycling through the four-step process [23]: Condensation by $\beta$-ketoacyl synthase (i.e., 3-oxoacyl-ACP synthase, mitochondrial; Oxsm); reduction by $\beta$-ketoacyl reductase 1 (i.e., 3-oxoacyl-ACP reductase), which consists of $\alpha$ subunit (i.e., 17 $\beta$-hydroxysteroid dehydrogenase 8, Hsd17b8) and $\beta$ subunit (i.e., carbonyl reductase 4, Cbr4) [24]; dehydration by 3-hydroxyacyl-CoA dehydratase 2 (Hacd2); and reduction by mitochondrial trans-2-enoyl-CoA reductase (Mecr). BMFA administration did not change the mRNA expression of these genes (Table 3). In addition, the genes that encode 
key enzymes in fatty acid biosynthesis, such as ATP citrate lyase (Acly), or mitochondrial glycerol-3-phosphate acyltransferase (Gpam), were not affected by BMFA administration.

Very long-chain fatty acids (VLCFAs) are elongated by cycling through the four-step process by the enzymes following their conversion to acyl-CoA [25]: Condensation by fatty acid elongase (Elovl1-7); reduction by functions as 3-ketoacyl-CoA reductase, which is known as $17 \beta$-hydroxysteroid dehydrogenase 12 (Hsd17b12); dehydration by 3-hydroxyacyl-CoA dehydratase (Hacd1-4); and reduction by 2,3-trans-enoyl-CoA reductase (Tecr) [25]. The microarray analysis showed that only Elovl7 mRNA significantly decreased among these genes.

Diacylglycerol $O$-acyltransferases (Dgat1 and Dgat2) synthesize triacylglycerol from diacylglycerol [9] (Figure 1). BMFA administration did not affected the Dgat mRNA levels (Table 3). The mRNA levels of free fatty acid receptor 2 (Ffar2) and fatty acid transport protein 3 (FATP3, encoded by the Slc27a3 gene) decreased in $o b / o b+B M F A$ mouse liver. In contrast, the mRNA levels of other fatty acid transporters (Slc27a2 and Slc27a4) did not significantly change.

Fourth, the genes related to fatty acid degradation were examined. Fatty acids degraded by oxidization of acyl-CoA to produce acetyl-CoA by various mechanisms, such as $\alpha$-oxidation, $\beta$-oxidation, and $\omega$-oxidation [26]. In mitochondria and peroxisomes, most fatty acids undergo $\beta$-oxidation by cycling through a four-step process (dehydrogenation, hydration of the double bond, dehydrogenation again, and thiolytic cleavage) [26]. In mitochondrial $\beta$-oxidation pathway, there are plasma membrane carnitine transporter (Slc25a29) and various enzymes: carnitine palmitoyltransferase 1 (Cpt1), carnitine/acylcarnitine translocase (Slc25a20), carnitine palmitoyltransferase (Cpt2), very long-chain acyl-CoA dehydrogenase (Acadvl), medium-chain acyl-CoA dehydrogenase (Acadm), short-chain acyl-CoA dehydrogenase (Acads), acyl-CoA dehydrogenase family member 9 (Acad9), 2-enoyl-CoA hydratase; longchain hydroxyacyl-CoA dehydrogenase, long-chain 3-ketoacyl-CoA thiolase, and mitochondrial trifunctional protein (MTP) [27]. As shown in Table 4, the expression of these enzymes was not affected by BMFA administration.

Table 4. Hepatic expression of mRNAs encoding the enzymes in fatty acid metabolism

\begin{tabular}{llr}
\hline Description (Abbreviation) & $\begin{array}{l}\text { Gene } \\
\text { symbol }\end{array}$ & $\begin{array}{l}\text { Signal ratio of } \\
\text { BMFA(+) vs. }\end{array}$ \\
\hline $\begin{array}{l}\text { Not } \text { changed: } \\
\text { Hydroxyacyl-CoA dehydrogenase/3-ketoacyl-CoA thiolase/ } \\
\text { enoyl-CoA hydratase (trifunctional protein), alpha subunit }\end{array}$ & Hadha & 0.832 \\
Acyl-CoA thioesterase 2 (ACOT2) & Acot2 & 0.859 \\
Acetoacetyl-CoA synthetase & Aacs & 0.893 \\
Acyl-CoA dehydrogenase, short chain (SCAD) & Acads & 0.911 \\
Acyl-CoA dehydrogenase, very long chain (VLCAD) & Acadvl & 0.925 \\
Acyl-CoA dehydrogenase family, member 9 & Acad9 & 0.952 \\
Acyl-CoA oxidase 2, branched chain & Acox2 & 0.959 \\
Carnitine palmitoyltransferase 2 (CPT2) & Cpt2 & 0.975 \\
Hydroxyacyl-CoA dehydrogenase/3-ketoacyl-CoA thiolase/ & Hadhb & 0.975 \\
enoyl-CoA hydratase (trifunctional protein), beta subunit & & 0.987 \\
Acyl-CoA thioesterase 8 (ACOT8) & Acot8 & 1.025 \\
Acyl-CoA thioesterase 6 (ACOT6) & Acot6 & 1.032 \\
Carnitine palmitoyltransferase 1a, liver & Cpt1a &
\end{tabular}


Acyl-CoA dehydrogenase family, member 9

Acad 9

1.052

Solute carrier family 25 (mitochondrial carnitine/acylcarnitine translocase), member 20

Acyl-CoA dehydrogenase, long-chain

Acyl-CoA thioesterase 7 (ACOT7)

Acyl-CoA thioesterase 12 (ACOT12)

Solute carrier family 25 (mitochondrial carrier, palmitoyl-

carnitine transporter), member 29

Acyl-CoA oxidase 1, palmitoyl

Acyl-CoA dehydrogenase, medium chain (MCAD)

Enoyl-CoA, hydratase/3-hydroxyacyl CoA dehydrogenase

Acyl-CoA thioesterase 4 (ACOT4)

Acyl-CoA dehydrogenase, short/branched chain

Acyl-CoA thioesterase 5 (ACOT5)

Increased:

Acyl-CoA thioesterase 3 (ACOT3)

Acot3

2.041

Cytochrome P450, family 4, subfamily a, polypeptide 31

Cур4a31

Cytochrome P450, family 4, subfamily a, polypeptide 10

Cyp 4 a10

${ }^{*}$ Signal ratios of probe sets in $o b / o b+$ BMFA vs. ob/ob-BMFA are expressed as the fold change, when the signal of $o b / o b-\mathrm{BMFA}$ was set to 1 .

There are several enzymes involved in peroxisomal $\beta$-oxidation, such as acyl-CoA oxidases (Acox1 and Acox2) [26]. Acyl-CoA thioesterases (ACOTs) are the enzymes that convert fatty acyl-CoAs to free fatty acids and CoA molecules [28,29]. After $\beta$-oxidation, fatty acids are metabolized in peroxisomes by ACOT3-6 and ACOT8 $[28,29]$. When the expression of these genes was examined, the levels of mRNAs encoding ACOT3 was significantly increased in $o b / o b+\mathrm{BMFA}$ mouse liver, whereas the levels of other many ACOT mRNAs were not affected (Table 4). In addition, BMFA administration significantly upregulated the expression of cytochrome P450 (Cyp4a31 and Cyp4a10) mRNAs in ob/ob mouse liver.

The genes related to cholesterol metabolism were also examined because dyslipidemia is observed in diabetes mellitus [5]. BMFA did not affect the levels of low-density lipoprotein receptor (Ldlr), apolipoprotein A1 (apoa1), or apolipoprotein E (apoe) mRNAs, with $\mathrm{BMFA}(+) /(-)$ signal ratios of $0.976,0.668$, and 1.118 , respectively.

\section{Effects of BMFA on the mRNA expression of the enzymes that catalyze glycolysis and gluconeogenesis}

Fifth, we investigated the genes that encode key enzymes in glycolysis and gluconeogenesis because it has been reported that fatty acid oxidation suppressed glycolysis and stimulated gluconeogenesis [30]. Indeed, the mRNA expression of mitochondrial phosphoenolpyruvate carboxykinase 2 (Pck2) and hexokinase 3 (Hk3) decreased in the liver of ob/ob+BMFA mice.

In contrast, BMFA administration did not affect the mRNA expression of several enzymes that catalyze both reactions in glycolysis and gluconeogenesis $\left({ }^{* *}\right.$ in Table 5). The mRNA expression levels of the other genes encoding many enzymes in glycolysis and gluconeogenesis were not changed by BMFA administration. Fructose-1,6-bisphosphatase 1 (Fbp1) is a pivotal enzyme in gluconeogenesis pathway and catalyzes the reverse reaction by phosphofructokinase, B-type (Pfkl) in glycolysis. The Fbp1 mRNA level was not affected by BMFA administration, 
whereas the mRNA encoding Fbp2 (isozyme of FBP1) was expressed at very low levels and was excluded (data not shown).

The microarray data indicated that the levels of mRNA's encoding all the enzymes in the citric acid cycle (classified by KEGG Pathway) were not affected by BMFA administration (data not shown).

Table 5. Hepatic expression of mRNAs encoding enzymes in glycolysis and gluconeogenesis

\begin{tabular}{|c|c|c|}
\hline Description & $\begin{array}{l}\text { Gene } \\
\text { symbol }\end{array}$ & $\begin{array}{l}\text { Signal ratio of } \\
\operatorname{BMFA}(+) v s .(-)^{*}\end{array}$ \\
\hline \multicolumn{3}{|l|}{ Glycolysis, Decreased: } \\
\hline Phosphoenolpyruvate carboxykinase 2 , mitochondrial & Pck2 & 0.432 \\
\hline Aldehyde dehydrogenase 3 family, member B1 & Aldh3bl & 0.488 \\
\hline Hexokinase 3 & $H k 3$ & 0.497 \\
\hline \multicolumn{3}{|l|}{ Glycolysis, Not changed: } \\
\hline UDP-glucose pyrophosphorylase 2 & $U g p 2$ & 0.750 \\
\hline Phosphofructokinase, liver, B-type & $P f k l$ & 0.724 \\
\hline Glucose phosphate isomerase $1^{* *}$ & Gpil & 0.738 \\
\hline Pyruvate kinase, liver and red blood cell & Pklr & 0.756 \\
\hline Glycerol-3-phosphate dehydrogenase 1, soluble & Gpdl & 0.772 \\
\hline Aldolase A, fructose-bisphosphate ${ }^{* *}$ & Aldoa & 0.796 \\
\hline Phosphoglycerate mutase $5^{* *}$ & Pgam5 & 0.826 \\
\hline Enolase 1 , alpha non-neuron ${ }^{* *}$ & Enol & 0.827 \\
\hline Glucokinase & Gck & 0.841 \\
\hline Glyceraldehyde-3-phosphate dehydrogenase ${ }^{* *}$ & Gapdh & 0.879 \\
\hline Triosephosphate isomerase $1^{* *}$ & Tpil & 0.924 \\
\hline Phosphoglycerate mutase $1^{* *}$ & Pgaml & 0.961 \\
\hline Aldolase B, fructose-bisphosphate ${ }^{* *}$ & Aldob & 0.993 \\
\hline Glycerol phosphate dehydrogenase 2, mitochondrial & $G p d 2$ & 1.052 \\
\hline Liver glycogen phosphorylase & Pygl & 1.054 \\
\hline Phosphoglycerate kinase $1^{* *}$ & Pgkl & 1.068 \\
\hline Glycogen synthase 2 & Gys2 & 1.112 \\
\hline Aldolase $\mathrm{C}$, fructose-bisphosphate ${ }^{* *}$ & Aldoc & 1.364 \\
\hline Phosphoenolpyruvate carboxykinase 1 , cytosolic & Pckl & 1.432 \\
\hline Glucose-6-phosphatase, catalytic & G6pc & 1.699 \\
\hline
\end{tabular}

* Signal ratios of probe sets in $o b / o b+$ BMFA vs. ob/ob-BMFA are expressed as the fold change, when the signal of $o b / o b-\mathrm{BMFA}$ was set to 1 .

** The enzyme catalyzes reactions in both glycolysis and gluconeogenesis.

The pyruvate dehydrogenase complex (PDC) catalyzes the oxidative decarboxylation of glucose-derived pyruvate to yield acetyl-CoA and ATP in mitochondria (Figure 1). PDC consists of four components: pyruvate dehydrogenase (E1), dihydrolipoamide $S$ acetyltransferase (E2), dihydrolipoamide dehydrogenase (E3), and the non-catalytic component $\mathrm{X}$ (i.e., E3-binding protein) [31]. These components are expressed in the liver: Pdhal (E1 component, subunit $\alpha$ ), Pdhb (E1 component, subunit $\beta$ ); Dlat (E2), Dld (E3), and Pdhx (E3-binding protein) genes. As shown in Table 6, the changes of the expression levels were not observed among these mRNAs. 
Table 6. Hepatic expression of mRNAs encoding pyruvate dehydrogenase complex and others

\begin{tabular}{llc}
\hline Description & $\begin{array}{l}\text { Gene } \\
\text { symbol }\end{array}$ & $\begin{array}{l}\text { Signal ratio of } \\
\text { BMFA(+) vs. }(-)^{*}\end{array}$ \\
\hline Gluconeogenesis, Not changed: & & \\
Pyruvate carboxylase & $P c x$ & 0.910 \\
Pyruvate dehydrogenase (lipoamide) beta (E1 beta subunit) & $P d h b$ & 0.914 \\
Lactate dehydrogenase A & Ldha & 0.955 \\
Fructose bisphosphatase 1 (= fructose-1,6-bisphosphatase 1) & Fbpl & 1.048 \\
Dihydrolipoamide $S$-acetyltransferase (E2 component) & Dlat & 1.135 \\
Pyruvate dehydrogenase E1 alpha 1 & Pdhal & 1.144 \\
Lactate dehydrogenase D & Ldhd & 1.292 \\
Dihydrolipoamide dehydrogenase (E3 component) & Dld & 1.425 \\
Phosphoenolpyruvate carboxykinase 1, cytosolic & $P c k l$ & 1.432 \\
Pyruvate dehydrogenase complex, component X & $P d h x$ & 1.513 \\
\hline
\end{tabular}

* Signal ratios of probe sets in $o b / o b+\mathrm{BMFA} v s . o b / o b-\mathrm{BMFA}$ are expressed as the fold change, when the signal of $o b / o b-\mathrm{BMFA}$ was set to 1.

Then, the expression of glucose transporter genes was investigated. Glucose transporters (GLUTs) are also designated as solute carrier family 2 (Slc2a). As shown in Table 7, the mRNA levels of most Slc2a members were not affected by BMFA administration, whereas Slc2a3 and Slc2a6 mRNAs were expressed at very low levels (data not shown).

Table 7. Hepatic expression of facilitated glucose transporter mRNAs

\begin{tabular}{llc}
\hline Description & $\begin{array}{l}\text { Gene } \\
\text { symbol }\end{array}$ & $\begin{array}{l}\text { Signal ratio of } \\
\text { BMFA }(+) \text { vs. }(-)^{*}\end{array}$ \\
\hline Not changed: & & \\
Solute carrier family 2 (facilitated glucose transporter), member 8 & Slc2a8 & 0.889 \\
Solute carrier family 2 (facilitated glucose transporter), member 9 & Slc2a9 & 0.896 \\
Solute carrier family 2 (facilitated glucose transporter), member 2 & Slc2a2 & 1.076 \\
Solute carrier family 2 (facilitated glucose transporter), member 1 & Slc2al & 1.184 \\
Solute carrier family 2 (facilitated glucose transporter), member 5 & Slc2a5 & 1.292 \\
Solute carrier family 2 (facilitated glucose transporter), member 4 & Slc2a4 & 1.305 \\
\hline
\end{tabular}

* Signal ratios of probe sets in $o b / o b+\mathrm{BMFA} v s . o b / o b-\mathrm{BMFA}$ are expressed as the fold change, when the signal of $o b / o b-\mathrm{BMFA}$ was set to 1 .

\section{Effects of BMFA on the mRNA expression of the genes involved in insulin resistance}

Finally, the genes that play an important role in insulin resistance were examined because proinflammatory cytokines are assumed to be partly responsible for the development of insulin resistance [5]. As shown in Table 8, the level of protein tyrosine phosphatase, non-receptor type 1 (Ptpn) mRNA did not change in $o b / o b+\mathrm{BMFA}$ mouse liver. This phosphatase (also known as protein tyrosine phosphatase 1B, PTB1B) antagonizes the insulin signaling pathway by dephosphorylating the insulin receptor [32]. BMFA administration did not affect the level of $O$-linked $N$-acetylglucosamine (GlcNAc) transferase OGT mRNA. This enzyme catalyzes the addition of a single GlcNAc in $O$-glycosidic linkage to serine or threonine residues, and its activity is implicated in insulin resistance in diabetes mellitus [33]. The level of insulin receptor mRNA was slightly, but not significantly, increased in $o b / o b+\mathrm{BMFA}$ mouse liver. 
Table 8. Hepatic expression of mRNAs involved in insulin resistance

\begin{tabular}{llc}
\hline Gene name (Abbreviation) & $\begin{array}{l}\text { Gene } \\
\text { symbol }\end{array}$ & $\begin{array}{l}\text { Signal ratio of } \\
\text { BMFA(+) vs. }(-)^{*}\end{array}$ \\
\hline Not changed: & & 0.822 \\
Protein tyrosine phosphatase, non-receptor type 1 (PTP1B) & Ptpn1 & 1.111 \\
O-Linked $N$-acetylglucosamine (GlcNAc) transferase (OGT) & Ogt & 1.207 \\
Insulin receptor & Insr & \\
\hline Increased: & & 2.099 \\
Peroxisome proliferative activated receptor $\gamma$, coactivator 1 $\alpha$ & Ppargcla & 2.144 \\
(PGC-1 $\alpha$ ) & Ppplr3c & \\
Protein phosphatase 1, regulatory (inhibitor) subunit 3C &
\end{tabular}

* Signal ratios of probe sets in ob/ob+BMFA $v s . o b / o b-$ BMFA are expressed as the fold change, when the signal of $o b / o b-\mathrm{BMFA}$ was set to 1 .

In contrast, BMFA administration increased the mRNA level of peroxisome proliferative activated receptor $\gamma$, coactivator $1 \alpha$ (Ppargc1a, PGC- $1 \alpha$ ) in $o b / o b+B M F A$ mouse liver. PGC$1 \alpha$ is a key factor for insulin-mediated suppression of glucose production [34]. BMFA reduced the level of mRNA encoding protein phosphatase 1, regulatory (inhibitor) subunit 3C (Ppp1r3c), which activates glycogen synthase by inhibiting glycogen phosphorylase activity. Among the genes classified in insulin resistance by KEGG Pathway, only Ppargc1a and Ppp1r3c mRNAs increased in $o b / o b+\mathrm{BMFA}$ mouse liver.

\section{DISCUSSION}

\section{Microarray analysis of mRNA expression in ob/ob mice}

Our microarray analysis of mRNA expression demonstrated that 2,632 genes (precisely, 2,632 probe sets) were affected by the EtOAc-soluble fraction of bitter melon fruit extract (i.e., BMFA) in the liver. A short-term effect of BMFA on hepatic gene expression was investigated using $o b / o b$ mice, a diabetes mellitus model [17].

Recently, high-throughput RNA sequencing (i.e., RNA-seq) was performed using $L e p r^{d b / d b}$ mice [35]. The leptin receptor gene is disrupted in these mice, which exhibit a similar phenotype to $o b / o b$ mice $\left(L e p^{o b / o b}\right)[7,17]$. The RNA-seq analysis indicated that the expression levels of 2,627 genes in the liver of $\operatorname{Lepr}^{d b / d b}$ mice were significantly changed compared with those of normal C57BL/6 mice [35]. The number of changed genes was comparable to our results.

\section{The mRNA expression of proinflammatory genes in BMFA-administered ob/ob mice}

The present study showed that BMFA downregulated proinflammatory genes, including cytokine and chemokine genes, in $o b / o b$ mouse liver (Tables 1 and 2). However, the repertoire of the proinflammatory genes affected by BMFA was different from those of previous reports using primary cultured rat hepatocytes. The hepatocytes mimic acute liver injury in response to the proinflammatory cytokine IL-1 $\beta$ [11]. The study showed that BMFA markedly decreased the levels of TNF, IL-6, and CCL20 mRNAs in IL-1 $\beta$-treated hepatocytes [14]. In contrast, BMFA administration slightly downregulated TNF mRNA expression, and IL-6 and CCL20 mRNAs were absent in $o b / o b$ mouse liver (data not shown).

It is known that NF- $\mathrm{BB}$ regulates the expression of many genes involved in inflammation and liver injury $[10,13]$. For example, limonin and obakunone, i.e., constituents from crude 
drugs of Japanese kampo medicine, reduced the expression of the $i N O S$ gene probably through $\mathrm{NF}-\kappa \mathrm{B}$ in IL-1 $\beta$-treated hepatocytes [18]. Interestingly, some genes induced by acute inflammation in the liver of endotoxemia model rats [19] were not decreased (e.g., iNOS, CRP, and LCN2) or absent (e.g., COX-2) in $o b / o b+\mathrm{BMFA}$ mouse liver. Due to chronic inflammation indirectly caused by leptin deficiency, a different repertoire of proinflammatory genes may be formed in $o b / o b$ mice, which may lead to different pathophysiological effects. Therefore, it is possible that NF- $\kappa \mathrm{B}$-mediated regulation in $o b / o b$ mouse liver is different from those in the hepatocytes and the liver of endotoxemia model rats.

\section{The mRNA expression of the enzymes that catalyze fatty acid pathways in BMFA- administered ob/ob mice}

The mRNA levels of many enzymes that catalyze fatty acid synthesis and metabolism were not significantly changed, although the expression of several genes was significantly affected by BMFA (Tables 3 and 4). As examples of the latter genes, the mRNA expression of genes involved in fatty acid synthesis including Ffar2 and FATP3/Slc27a3 was downregulated, whereas the mRNA expression of genes involved in fatty acid metabolism such as ACOT3 was upregulated in the liver of $o b / o b+\mathrm{BMFA}$ mice.

It is possible that these effects of BMFA administration may improve lipid accumulation by the involvement of the Ffar2 and FATP3/Slc27a3 genes. Indeed, when a high-fat diet was fed to Ffar2-deficient mice, these mice had lower body fat mass and improved glucose control [36]. Overflow of fatty acids is thought to drive many obesity-associated diseases [37]. Because FATP3/Slc27a3 is responsible for the uptake and activation of long-chain fatty acids, it might regulate overflow. Additionally, many ACOTs metabolize lipids to fatty acids [28,29], and the fatty acids in liver induce inflammation [5,6].

In hepatocytes, fatty acids are stored as triglyceride, whose amount correlates with net production of fatty acid via several pathways. The quantity of a product generally correlates with both quantity and activity of an enzyme, and the resultant product is used as a substrate of the next enzyme. Therefore, the quantity of the final product (triglyceride in this case) is determined by both quantity and activity of all the enzymes in the pathway. Furthermore, insulin and other signaling molecules tightly regulate production of enzymes in the pathways [9]. Taken together, microarray data (i.e., expression of enzyme mRNAs) obtained in this study are insufficient to estimate net production of fatty acid and triglyceride.

It is necessary to measure the amount of triglyceride in the liver. Because hyperlipidemia is observed in $o b / o b$ mice [17], it is probable that triglyceride accumulates in the liver. Obesity, insulin resistance, and diabetes mellitus are closely linked to excessive lipid accumulation in the liver, and lipid accumulation is also observed in NAFLD, including non-alcoholic steatohepatitis (NASH) [9,38]. More studies using animals are required to examine whether BMFA improves hepatic lipid accumulation.

\section{The mRNA expression of the enzymes that catalyze glucose and glycogen pathways in BMFA-administered ob/ob mice}

The microarray data clearly indicated that BMFA administration did not affect the expression of the genes that encode most key enzymes in glycolysis and gluconeogenesis, as well as glycogen pathways (Tables 5, 6). Although most genes were highly expressed in the liver (data not shown), only the mRNA expression levels of mitochondrial phosphoenolpyruvate carboxykinase 2 (Pck2), aldehyde dehydrogenase 3 family, member B1 (Aldh3b1), and 
hexokinase 3 (Hk3) were significantly downregulated in $o b / o b+\mathrm{BMFA}$ mouse liver. The expression of glucose transporter (Slc2a) mRNAs was not significantly changed by BMFA administration (Table 7).

Because obesity-induced hyperglycemia is observed in $o b / o b$ mice [17], dysregulation of glucose and glycogen pathways are expected to be present. Similarly, to the complex lipid pathways, it is difficult to estimate net production of glucose and glycogen by the microarray data. Furthermore, enzyme activity is modulated by various mechanisms. For example, glucokinase (Gck), which converts glucose to glucose-6-phosphate in glycolysis, plays a key role to store glucose as glycogen. Insulin and glucagon modulate activity of this enzyme [8]. It remains to be studied to clarify the dysregulation of glucose and glycogen pathways.

\section{The mRNA expression of the genes involved in insulin resistance in BMFA-administered ob/ob mice}

When the genes that play an important role in insulin resistance were analyzed (Table 8), the PGC- $1 \alpha$ mRNA level was significantly upregulated in $o b / o b+B M F A$ mice, although BMFA did not significantly change the levels of Ogt mRNA or insulin receptor mRNA. PGC-1 $\alpha$ plays critical roles in insulin-mediated suppression of glucose production by promoting gluconeogenesis [34]. Because PGC-1 $\alpha$ defines the precise insulin signal in hepatocytes in response to fasting and feeding, a reduction in PGC- $1 \alpha$ is associated with hepatic insulin resistance [34,39]. Therefore, it is possible that PGC-1 $\alpha$ may be responsible for the action of EtOAc-soluble constituents in BMFA.

Our previous study suggested that cucurbitane-type triterpenoids are included in BMFA, i.e., EtOAc-soluble fraction from bitter melon fruit extract [14]. It is speculated that these triterpenoids may suppress the NF- $\mathrm{BB}$ signaling pathway, leading to the improvement of inflammation and metabolic dysregulation in the liver. Indeed, bitter melon fruit has been used as an antidiabetic drug in traditional Indonesian medicine. If cucurbitane-type triterpenoids possess hypoglycemic or antidiabetic activity, they might provide a lead compound to develop a new drug for obesity, NAFLD, NASH, and diabetes mellitus.

Although only the leptin gene is disrupted in the $o b / o b$ mouse, this deficiency provokes a complex combination of metabolic dysregulation that causes a variety of phenotypes, such as hyperglycemia, hyperlipidemia, and obesity. Involvement of the roles of many enzymes in obesity and insulin resistance has been pointed out. For example, glycerol-3-phosphate acyltransferase (e.g., Gpam), which catalyzes the first step of synthesis of triacylglycerol, plays a critical role in the development of insulin resistance [40]. Further investigation of the BMFAadministered $o b / o b$ mice is necessary to examine antidiabetic effects of BMFA at a molecular level. Recently, we reported about them elsewhere [41].

\section{CONCLUSION}

The EtOAc-soluble fraction of a bitter melon fruit extract was administered to $o b / o b$ mice, and short-term changes in the mRNA expression pattern of the liver were analyzed using microarrays. The fraction (BMFA) downregulated the genes involved in inflammation, whereas it did not change the expression of many mRNAs encoding the enzymes in fatty acid and glucose pathways in $o b / o b$ mouse liver. In addition, BMFA increased the PGC- $1 \alpha$ mRNA level. It is expected that EtOAc-soluble constituents may possess anti-inflammatory activity, which should be examined in the future. 
Abbreviations: TNF, tumor necrosis factor; NF- $\kappa \mathrm{B}$, nuclear factor- $\kappa \mathrm{B}$; IL, interleukin; EtOAc, ethyl acetate; KEGG, Kyoto Encyclopedia of Genes and Genomes; BMFA, ethyl acetatesoluble fraction of bitter melon fruit extract; CCL, chemokine $\mathrm{C}-\mathrm{C}$ motif ligand; CoA, Coenzyme A; ACP, acyl-acyl carrier protein; ACOT, acyl-Coenzyme A thioesterase; Slc, solute carrier; PGC-1 $\alpha$, peroxisome proliferative activated receptor $\gamma$, coactivator $1 \alpha$.

Acknowledgments and funding: We thank Ms. Noriko Kanazawa for her secretarial assistance. D.R.D. was supported by the Ministry of Education, Culture, Sports, Science and Technology (MEXT), Japan, for the research and the stay in Japan. This work was supported in part by the Asia-Japan Research Institute of Ritsumeikan of the Asia-Japan Research Organization, Ritsumeikan University (Ibaraki, Osaka, Japan).

Authors' contributions: DRD and TI performed the experiments, data collection, and statistical analysis and drafted the manuscript as graduate students. TO analyzed the data and provided advice. EM and MN participated in the design of the study, supervised the study, and provided oversight in the drafting of the manuscript.

Competing Interests: D.R.D. and T.I. carried out this study as graduate students of the Graduate School of Life Sciences, Ritsumeikan University.

\section{REFERENCES}

1. Chen Q, Li ETS: Reduced adiposity in bitter melon (Momordica charantia) fed rats is associated with lower tissue triglyceride and higher plasma catecholamines. Br J Nutr. 2005 93: 747-754.

2. Chaturvedi P: Antidiabetic potentials of Momordica charantia: multiple mechanisms behind the effects. J Med Food. 2012 15: 101-107.

3. Paniagua JA: Nutrition, insulin resistance and dysfunctional adipose tissue determine the different components of metabolic syndrome. World J Diabetes. 2016 7: 483-514.

4. Guh DP, Zhang W, Bansback N, Amarsi Z, Birmingham CL, Anis AH: The incidence of co-morbidities related to obesity and overweight: a systematic review and metaanalysis. BMC Public Health. 2009 9: 1-20.

5. Schofield JD, Liu Y, Rao-Balakrishna P, Malik RA, Soran H: Diabetes dyslipidemia. Diabetes Ther. 2016 7: 203-219.

6. Milagro FI, Campión J, Martínez JA: Weight gain induced by high-fat feeding involves increased liver oxidative stress. Obesity (Silver Spring). 2006 14: 1118-1123

7. Wasim M, Awan FR, Najam SS, Khan AR, Khan HN: Role of leptin deficiency, inefficiency, and leptin receptors in obesity. Biochem Genet. 2016 54: 565-572.

8. Petersen MC, Vatner DF, Shulman GI: Regulation of hepatic glucose metabolism in health and disease. Nat Rev Endocrinol. 2017 13: 572-587.

9. Alves-Bezerra M, Cohen DE: Triglyceride metabolism in the liver. Compr Physiol. 2019 8: 1-8.

10. Tacke F, Luedde T, Trautwein C: Inflammatory pathways in liver homeostasis and liver injury. Clin Rev Allergy Immunol. 2009 36: 4-12. 
11. Kitade H, Sakitani K, Inoue K, Masu Y, Kawada N, Hiramatsu Y, Kamiyama Y, et al.: Interleukin $1 \beta$ markedly stimulates nitric oxide formation in the absence of other cytokines or lipopolysaccharide in primary cultured rat hepatocytes but not in Kupffer cells. Hepatology. 1996 23: 797-802.

12. Yoshigai E, Hara T, Inaba H, Hashimoto I, Tanaka Y, Kaibori M, Kimura T, et al.: Interleukin-1 $\beta$ induces tumor necrosis factor- $\alpha$ secretion from rat hepatocytes. Hepatol Res. 2014 44: 571-583.

13. Karin M: NF- $\kappa B$ as a critical link between inflammation and cancer. Cold Spring Harb Perspect Biol. 2014 1: a000141.

14. Dwijayanti DR., Okuyama T, Okumura T, Ikeya Y, Nishizawa M: The antiinflammatory effects of Indonesian and Japanese bitter melon (Momordica charantia L.) fruit extracts on interleukin-1 $\beta$-treated hepatocytes. Functional Foods in Health and Disease. 2019 9: 16-33.

15. Hotamisligil GS: Inflammatory pathways and insulin action. Int J Obes Relat Metab Disord. 2003 27: S53-S55.

16. Abedini A, Shoelson SE: Inflammation and obesity: STAMPing out insulin resistance? Immunol Cell Biol. 2007 85: 399-400.

17. King AJ: The use of animal models in diabetes research. Br J Pharmacol. 2012 166: 877-894.

18. Fujii A, Okuyama T, Wakame K, Okumura T, Ikeya Y, Nishizawa, M: Identification of anti-inflammatory constituents in Phellodendri Cortex and Coptidis Rhizoma by monitoring the suppression of nitric oxide production. J Nat Med. 2017 71: 745-756.

19. Okuyama T, Nakatake R, Kaibori M, Okumura T, Kon M, Nishizawa M: A sense oligonucleotide to inducible nitric oxide synthase mRNA increases the survival rate of rats in septic shock. Nitric Oxide. 2018 72: 32-40.

20. Inaba H, Yoshigai E, Okuyama T, Murakoshi M, Sugiyama K, Nishino H, Nishizawa M: Antipyretic analgesic drugs have different mechanisms for regulation of the expression of inducible nitric oxide synthase in hepatocytes and macrophages. Nitric Oxide. 2015 44: 61-70.

21. Sproston NR, Ashworth JJ: Role of C-reactive protein at sites of inflammation and infection. Front Immunol. 2018 9: 754.

22. Moschen AR, Adolph TE, Gerner RR, Wieser V, Tilg H: Lipocalin-2: a master mediator of intestinal and metabolic inflammation. Trends Endocrinol Metab. 2017 28: 388-397.

23. Masud AJ, Kastaniotis AJ, Rahman MT, Autio KJ, Hiltunen JK: Mitochondrial acyl carrier protein (ACP) at the interface of metabolic state sensing and mitochondrial function. Biochim Biophys Acta Mol Cell Res. 2019 1866: 118540.

24. Chen Z, Kastaniotis AJ, Miinalainen IJ, Rajaram V, Wierenga RK, Hiltunen JK: 17 $\beta$ hydroxysteroid dehydrogenase type 8 and carbonyl reductase type 4 assemble as a ketoacyl reductase of human mitochondrial FAS. FASEB J. 2009 23: 3682-3691.

25. Kihara A: Very long-chain fatty acids: elongation, physiology and related disorders. J Biochem. 2012 152: 387-395. 
26. Wanders RJ: Peroxisomes, lipid metabolism, and peroxisomal disorders. Mol Genet Metab. 2004 83: 16-27.

27. Kompare M, Rizzo WB: Mitochondrial fatty-acid oxidation disorders. Semin Pediatr Neurol. 2008 15:140-149.

28. Tillander V, Alexson SEH, Cohen DE: Deactivating Fatty Acids: Acyl-CoA Thioesterase-Mediated Control of Lipid Metabolism. Trends Endocrinol Metab. 2017 28: 473-484.

29. Hunt MC, Siponen MI, Alexson SE: The emerging role of acyl-CoA thioesterases and acyltransferases in regulating peroxisomal lipid metabolism. Biochim Biophys Acta. 2012 1822: 1397-1410.

30. Randle PJ: Regulatory interactions between lipids and carbohydrates: the glucose fatty acid cycle after 35 years. Diabetes Metab Rev. 1999 14: 263-283.

31. Brautigam CA, Wynn RM, Chuang JL, Chuang DT: Subunit and catalytic component stoichiometries of an in vitro reconstituted human pyruvate dehydrogenase complex. J Biol Chem. 2009 284: 13086-13098.

32. Klomann SD, Mueller AS, Pallauf J, Krawinkel MB: Antidiabetic effects of bitter gourd extracts in insulin-resistant $d b / d b$ mice. Br J Nutr. 2010 104: 1613-1620.

33. Ruan HB, Singh JP, Li MD, Wu J, Yang X: Cracking the $O$-GlcNAc code in metabolism. Trends Endocrinol Metab. 2013 24: 301-309.

34. Besseiche A, Riveline JP, Gautier JF, Breant B, Blondeau B: Metabolic roles of PGC$1 \alpha$ and its implications for type 2 diabetes. Diabetes Metab. 2015 41: 347-357.

35. Zhang F, Xu X, Zhang Y, Zhou B, He Z, Zhai Q: Gene expression profile analysis of type 2 diabetic mouse liver. PLoS One. 2013 8: 1-11.

36. Bjursell M, Admyre T, Goransson M, Marley AE, Smith DM, Oscarsson J, BohloolyYM: Improved glucose control and reduced body fat mass in free fatty acid receptor 2-deficient mice fed a high-fat diet. Am J Physiol Endocrinol Metab. 2011 300: E211E220.

37. Kazantzis M, Stahl A: Fatty acid transport proteins, implications in physiology and disease. Biochim Biophys Acta. 2012 1821: 852-857.

38. Finck BN: Targeting metabolism, insulin resistance, and diabetes to treat nonalcoholic steatohepatitis. Diabetes. 2018 67: 2485-2493.

39. Besse-Patin A, Jeromson S, Levesque-Damphousse P, Secco B, Laplante M, Estall JL: PGC1A regulates the IRS1:IRS2 ratio during fasting to influence hepatic metabolism downstream of insulin. Proc Natl Acad Sci USA. 2019 116: 4285-4290.

40. Yu J, Loh K, Song ZY, Yang HQ, Zhang Y, Lin S: Update on glycerol-3-phosphate acyltransferases: the roles in the development of insulin resistance. Nutr Diabetes. 2018 8: 34.

41. Dwijayanti DR, Shimada T, Ishii T, Okuyama T, Ikeya Y, Mukai E, Nishizawa M: Bitter melon fruit extract has a hypoglycemic effect and reduces hepatic lipid accumulation in $o b / o b$ mice. Phytotherapy Research, in press. DOI: 10.1002/ptr.6600 